\title{
Constructing a pipeline for genome variant / gene functioning hybrid prioritization: a case study of type II diabetes
}

\author{
Irina Kolesnikova \\ LLC NCGI, Novosibirsk, Russia \\ i.kolesnikova@mygenetics.ru
}

\author{
Valery Polunovsky \\ LLC NCGI, Novosibirsk, Russia \\ valeriy.polunovskiy@mygenetics.ru
}

\author{
Konstantin Gunbin \\ ICG SB RAS, Novosibirsk, Russia \\ NSU, Novosibirsk, Russia \\ genkvg@bionet.nsc.ru
}

\begin{abstract}
According to recently proposed "omnigenic model" the genes implicated in complex diseases determination can be divided into core genes and peripheral genes. Mutations in core genes directly affect disease development, while mutations in peripheral genes can only indirectly modulate disease risk. In this study in order to discriminate core genes and their major regulators, we hierarchically combine genome variant prioritization with the prioritization of genes and target tissues.
\end{abstract}

Keywords - hierarchical prioritization, genome variant, gene functioning, type II diabetes

\section{Motivation and aim}

One of the major challenge in the interpretation of GWAS results is the only modest or even very weak contribution of important loci to the total heritability of complex diseases. Earlier an "omnigenic model" proposed in which genes implicated in complex diseases determination divided into core genes and peripheral ones according to the gene network of trait [1]. It is of big importance that modern theoretical investigations uncover that biological reason of modest or weak implication of variations in core genes to the total heritability of complex traits is primary rooted in strict negative selection on core genes [2]. Therefore, it is tempting to analyze GWAS data taking into consideration modern views on the gene network basis of complex traits and on the evolutionary reasons of effect sizes distribution across variants uncovered by GWAS investigations.

\section{Methods}

In order to do so, we construct pipeline composed of hierarchically combination of prioritizations procedures starting from genome variant prioritization and ending by target tissues prioritization.

First, we focused on the most stable and most studied single nucleotide variants (SNVs) that are uniquely annotated starting from the NCBI dbSNP v. 150, stable SNVs (SSNVs). We performed a frequency and a functional filtering of the selected SSNVs. Frequency filtering of SSNVs was made using the ANNOVAR platform.

Functional filtering of SSNVs was the following: the effect of each SSNVs located in the coding regions or splicing sites must have been thorough tested on protein structure and function; the effect of each regulatory SSNVs must have the experimental and consistent confirmation of biological significance. All SSNVs should be characterized by an evolutionary and functional conservation, located inside (and not between) linkage disequilibrium blocks for the Asian and European populations, and have well-characterized allele frequencies in the public databases. In order to prioritize selected SSNVs in each trait under analysis, we have developed a consistency function that takes into account the above described characteristics types of genome variants and based on nonparametric statistics and randomization procedures.

Second, we prioritize genes containing SSNVs (or regulated by upstream regions containing SSNVs) according to (1) the characteristics of gene haploinsufficiency, (2) the gene networks related to the standardized vocabularies of phenotypic abnormalities from Human Phenotype Ontology (HPO) resource and vocabularies of medical diagnoses from International Classification of Diseases (ICD) v. 10, (3) the position of the gene in the topology of these gene networks, (4) the relation of the gene to the known thorough tested network modules of complex diseases, and (5) the pleiotropy of gene across various complex traits. We developed normalization function that reconcile the above-mentioned gene characteristics.

Third, if the determination of complex disease dispersed through various tissues and organs, we assigned a tissue score based on the position of genes in the network topologies and on the level of their tissue-specific expression.

In our work, we used GeneAtlas [3] as the main GWAS data source. We choose GeneAtlas because 1) it is the only resource that has largest number of SNVs ever (more than $34 \mathrm{M}$ of all SNVs including imputed ones); 2) it deposited GWAS data for more than 650 clinical and more than 110 normal human traits; 3) it is based on an unprecedented number $(>450,000)$ of experimentally genotyped, unrelated individuals. These unique GeneAtlas features allowed [3]: 1) to obtain the highest heritability of the studied traits (significant reduction of the "missing heritability" artifact), 2) to get rid of the Winner's Curse artifact overestimating the strength of SNV effect size on small samples.

\section{Results}

A hierarchical prioritization pipeline for searching and prioritizing genes and SNVs associated with a risk to diseases was developed. We check our pipeline on wellinvestigated type II diabetes. Significant SNPs were selected in more than 100 genes / regulatory regions strongly associated with type II diabetes, its complications and comorbid diseases (Table 1).

\begin{tabular}{|c|c|c|}
\hline NOS3 & 6.752 & 42.753 \\
\hline MC4R & 7.392 & 40.975 \\
\hline TERF2IP & 3.069 & 39.417 \\
\hline NEUROG3 & 9.068 & 38.841 \\
\hline TUFM & 10.113 & 37.669 \\
\hline
\end{tabular}


The use of information on the gene networks structure and tissue-/organ- specificity of gene expression clarifies substantially our initial SSNVs prioritization, however, analysis of all GeneAtlas traits is significantly complicated by poor compliance between ICD and HPO vocabularies.

TABLE 1. THE TOP-30 LIST OF PRIORITIZED GENES ASSOCIATED WITH TYPE II DIABETES AND RELATED TO PANCREAS FUNCTIONING

\begin{tabular}{|c|c|c|}
\hline Gene name & $\begin{array}{c}\text { Normalised } \\
\text { functioning score in } \\
\text { pancreas }\end{array}$ & Composite score \\
\hline MICB & 13.457 & 195.911 \\
\hline EHMT2 & 5.167 & 180.766 \\
\hline KCNK3 & 7.713 & 146.456 \\
\hline AGT & 6.367 & 119.92 \\
\hline APOE & 8.236 & 101.094 \\
\hline ZFP36L2 & 12.935 & 90.303 \\
\hline CTRB1 & 14.31 & 75.996 \\
\hline MAP2K7 & 7.863 & 74.064 \\
\hline OPRL1 & 3.209 & 62.166 \\
\hline SPRY2 & 5.893 & 58.241 \\
\hline REL & 7.016 & 50.483 \\
\hline HLA-DPB1 & 8.152 & 47.214 \\
\hline LDLR & 10.696 & 45.945 \\
\hline HLA-C & 13.271 & 45.123 \\
\hline & & \\
\hline
\end{tabular}

\begin{tabular}{|c|c|c|}
\hline TAP1 & 3.554 & 33.596 \\
\hline HLA-DPA1 & 9.605 & 32.237 \\
\hline LIPC & 4.019 & 23.976 \\
\hline ATP2A1 & 5.899 & 23.589 \\
\hline HNF1A & 7.84 & 23.197 \\
\hline DYNC2LI1 & 2.162 & 22.966 \\
\hline MFF & 5.807 & 22.501 \\
\hline ANK1 & 6.117 & 21.774 \\
\hline SH3YL1 & 5.179 & 21.561 \\
\hline TCEA2 & 3.132 & 21.461 \\
\hline ACP5 & 3.637 & 20.822 \\
\hline
\end{tabular}

References

[1] Liu X, Li YI, Pritchard JK. (2019) Trans Effects on Gene Expression Can Drive Omnigenic Inheritance. Cell. 177(4):1022-1034.e6.

[2] O'Connor LJ, et al. (2019) Extreme Polygenicity of Complex Traits Is Explained by Negative Selection. Am J Hum Genet. 105(3):456-476.

[3] Canela-Xandri O, Rawlik K, Tenesa A. (2018) An atlas of genetic associations in UK Biobank. Nat Genet. 50(11):1593-1599. 